\title{
Comités de ética en investigación con seres humanos: relevancia actual en Colombia Experiencia de la Fundación Santa Fe de Bogotá
}

\author{
Human research ethics committees: their \\ relevance in Colombia today \\ The experience at the Fundación Santa Fe de Bogotá \\ Paula Prieto • Bogotá, D.C. (Colombia)
}

\section{Resumen}

En este artículo se revisan los antecedentes históricos que propiciaron la aparición de los Comités de Ética de Investigación con Seres Humanos en el mundo, su fundamentación ética, la principal reglamentación a nivel internacional y la existente en Colombia. Enmarcado en ese contexto y por la relevancia que tienen en la actualidad, se hace un recuento de la creación, el desarrollo y las actividades del Comité de Ética en la Investigación de la Fundación Santa Fe de Bogotá. Este comité, creado en 1983, ha ejercido sus funciones durante 25 años con la búsqueda permanente de la excelencia en todas sus actividades dirigidas a la protección de la dignidad, los derechos y el bienestar de los sujetos que participan en investigaciones biomédicas y sociales (Acta Med Colomb 2011; 36: 98-104).

Palabras clave: ética, investigación, seres humanos, comités, reglamentación, Colombia, Fundación Santa Fe de Bogotá.

\begin{abstract} and social research (Acta Med Colomb 2011; 36: 98-104). Fe de Bogotá.

\section{Introducción}

Enmarcado dentro de los antecedentes históricos que motivaron la aparición de los comités de ética de investigación con seres humanos en el mundo, su relevancia actual y su justificación ética y legal, el objetivo de este artículo es hacer un recuento cronológico de las actividades del Comité de Ética de Investigación con Seres Humanos (CEI con SH) de la Fundación Santa Fe de Bogotá, desde sus inicios, su desarrollo a lo largo de los años y su estructura presente.
\end{abstract}

This article reviews the historical background that led to the appearance of Human Research Ethics Committees the world, their ethical foundations, the main international regulations, and the ones existing in Colombia. Framed in this context and its relevance today, an account is presented of the creation, development, and activities of the Committee of the Fundación Santa Fe de Bogotá. This committee, created in 1983, has held office for 25 years with the ongoing pursuit of excellence in all activities aimed at protecting the dignity, rights, and welfare of subjects participating in biomedical

Keywords: ethics, research, human beings, committees, regulations, Colombia, Fundación Santa
Dra. Paula Prieto Martínez: Magíster en Bioética, Pontificia Universidad Javeriana, Miembro del Grupo de Trabajo del Centro de Humanismo y Bioética de la Fundación Santa Fe de Bogotá. Miembro del Comité de Ética de Investigaciones con Seres Humanos de la Fundación Santa Fe de Bogotá. Bogotá, D.C. (Colombia).

Correspondencia: Dra. Paula Prieto.

E-mail: paula_prietom@hotmail.com Recibido: 05/IV/2011 Aceptado: 07/VI/2011

\section{Antecedentes de la investigación que involucra seres humanos}

Aspectos históricos, fundamentación ética, importancia de los comités de ética y reglamentaciones relevantes
Históricamente se han hecho y se seguirán haciendo investigaciones involucrando seres humanos en aras de obtener conocimientos, que permitan el avance de la ciencia. Los médicos y científicos han querido saber más acerca de la fisiología y la patología del ser humano y han usado diferentes métodos para obtener dicha información, para mejorar la salud de los seres humanos y por consiguiente su calidad de vida. En la actualidad se presenta un reto al tratar de mantener el equilibrio entre el avance de la ciencia para mejorar las condiciones de vida y la protección de los derechos de los sujetos que participan en investigaciones.

La práctica de investigar con seres humanos es tan antigua como la medicina pero la preocupación por las consecuencias y por la protección de los sujetos participantes es un fenómeno reciente. Aunque no siempre fue una investigación 
formal como la que se realiza en la actualidad, desde el inicio de la medicina se realizaban actos unidos a la práctica clínica, como la analogía, que junto con la enfermedad misma y el azar, aumentaban los conocimientos científicos, pero siempre buscando la mejoría del paciente. La intención era, por lo tanto, beneficente y se asumía que las cualidades morales de los médicos no necesitaban ser vigiladas, pues se daba por sentado su idoneidad, su comportamiento ético y la autorregulación entre los profesionales (1).

A partir del final del siglo XIX y el inicio del siglo XX, siguiendo los cambios modernos en la forma de abordar la realidad en las áreas científicas donde se imponía validar los conocimientos obtenidos y junto con los avances de la estadística, se exigió pasar de los experimentos casuales de la etapa clásica anterior a los experimentos diseñados introduciendo la idea de diseño experimental (1).

En las primeras décadas del siglo XX se llevaron a cabo numerosos experimentos clínicos con seres humanos, que a pesar de haber dado el consentimiento para participar, fueron abusivos por los riesgos que implicaban y por no estar claro que el criterio ético que los guiaba fuera la beneficencia del paciente. Se justificaban experimentos que causaban daños inequívocos, incluso la muerte, en la medida que los sujetos hubieran dado su consentimiento voluntario. Se presentó un adelanto en el campo de la autonomía del paciente pero un retroceso en la beneficencia (1).

Sin embargo, los abusos cometidos pusieron en tela de juicio el avance de la autonomía cuando las investigaciones se desarrollaron, en la mayoría de los casos, en hospitales de beneficencia, con pacientes pobres o marginales, con escasa instrucción y sin evaluar de manera adecuada los riesgos frente a los beneficios (2).

Ante esta situación surgió la necesidad de una regulación de la experimentación. En el año 1931 en Alemania se promulgó una ley que daba importancia fundamental al respeto de la autonomía al exigir la obtención de consentimiento informado, tanto en investigaciones sobre medicamentos como en experimentación con humanos y en 1935 en los Estados Unidos se permitió la experimentación con seres humanos si daban su consentimiento (1).

A pesar de lo anterior, durante la Segunda Guerra Mundial, investigadores alemanes, muchos de ellos médicos, realizaron numerosos procedimientos en campos de concentración con poblaciones obviamente vulnerables, en especial prisioneros judíos y gitanos, pero también prisioneros políticos y otros. Dentro de las experimentaciones más conocidas, están aquellas sobre hipotermia, donde se probaban mecanismos para producir la muerte por congelamiento, observando los cambios fisiológicos que se producían hasta la muerte. Hay reportes sobre las investigaciones realizadas para conocer los efectos de venenos o de inyecciones intravenosas de gasolina (3).

Dada la libertad para actuar que prevalecía entre los científicos e investigadores, y la confianza en su capacidad moral para la práctica clínica y la realización de las investi- gaciones, llegando a considerar innecesario e inconveniente legislar sobre estas situaciones, no sorprende lo escandaloso $\mathrm{y}$ aterrador que fueron los testimonios sobre estas atrocidades cometidas durante la Segunda Guerra Mundial. No deja de ser contradictorio y hasta irónico ya que la legislación alemana de 1931 enfatizaba la importancia del consentimiento informado, del diseño adecuado de los estudios y de la protección especial a los sujetos vulnerables. Estaba totalmente prohibido experimentar con sujetos moribundos (3).

Las experiencias anteriores sentaron las bases para la promulgación del Código de Nüremberg, primer documento internacional regulatorio sobre la experimentación en seres humanos en 1947 (4). El documento, cuyo objetivo es proteger la integridad de la persona que se somete a un experimento, estipula las condiciones necesarias para la realización de trabajos de investigación con seres humanos, poniendo especial relieve en el consentimiento voluntario de las personas participantes de los estudios, en los riesgos justificados, en los beneficios anticipados, en la disminución del sufrimiento físico y mental de los sujetos, en el retiro de los mismos en el momento deseado sin sufrir represalias de ningún tipo y por último, que las investigaciones sean desarrolladas por científicos idóneos y capacitados (1).

En 1964, la Asociación Médica Mundial, en Helsinki, promulgó la Declaración de Helsinki en la que aparecen en forma clara dos capítulos, uno dedicado a la investigación médica combinada con asistencia profesional y el otro referente a la investigación biomédica no terapéutica. Esta declaración ha tenido considerable influencia en la formulación de las legislaciones y de los códigos de conducta internacionales y nacionales. La última revisión fue realizada en Corea en el año 2008 (5).

Se trata de un planteamiento internacional de gran alcance. Fundamentalmente, establece pautas éticas para los médicos que realizan investigación biomédica clínica y no clínica, y estipula, entre sus diversas reglas, la obligatoriedad de obtener el consentimiento informado de las personas que participan en la investigación. Adapta sus principios a la ética de la profesión médica, introduce las nociones de riesgo-beneficio para los sujetos experimentales y afirma la necesidad de la revisión de los protocolos de investigación por parte de un comité independiente de los investigadores, lo que ha sido mantenido y reforzado en la última revisión (5).

A pesar de lo anterior y de la promulgación del Código de Nüremberg, las injusticias continuaron como lo evidenció Beecher al publicar en 1966 un artículo en el New England Journal of Medicine, Ethics and Clinical Research, donde denunciaba investigaciones concretas con serios problemas éticos e insistía en el juicio correcto y virtuoso de los científicos más que en las reglamentaciones rígidas. Sus comentarios influyeron y sensibilizaron al público norteamericano y más aun al darse a conocer a la opinión pública otras arbitrariedades cometidas, como el caso ocurrido en el Hospital Judío de Enfermedades Crónicas en Brooklyn, Nueva York 
en 1963, donde se inyectaron por vía subcutánea células cancerosas a 22 pacientes ancianos sin su consentimiento para conocer la respuesta inmunológica; o el ocurrido en la Escuela Estatal de Willowbrook para niños, muchos de ellos con discapacidad mental, en Staten Island, Nueva York, donde con el fin de buscar una vacuna contra la hepatitis un grupo de médicos inició en 1956 un estudio infectando deliberadamente de hepatitis a los niños recién ingresados, asumiendo que de todas maneras se infectarían al estar en el centro por el hacinamiento en que se encontraban y que sólo seguirían el curso natural de la enfermedad (1).

Para tratar de controlar los abusos e irregularidades que se continuaban presentando, la agencia federal norteamericana conocida como FDA ( U.S. Food and Drug Administration) en 1963 hizo público un nuevo reglamento que había de regir la experimentación con nuevos fármacos y los NIH (National Institutes of Health) y el Departamento de Salud y Bienestar aplicaron criterios parecidos a toda la investigación biomédica; en 1966 publicaron unas normas en las que obligaban que los protocolos fueran revisados por un comité de la institución, dando origen a los Comités de Ética de Investigación (1).

Se había establecido en la Declaración de Helsinki, y aquí se reiteró, la importancia de la revisión de los protocolos de investigación por parte de un comité independiente y entre los puntos que se debían considerar y revisar estaba la adecuada obtención del consentimiento informado y la relación entre riesgo-beneficio para tener en cuenta los derechos y bienestar del paciente (2).

A principios de 1932 el Servicio de Salud Publica de los Estados Unidos inició un estudio para seguir el curso natural de la sífilis en una población de raza negra en el Instituto de Tuskegee (Condado de Macon, Alabama), incluyendo a cuatrocientos hombres con diagnóstico de sífilis en comparación con otros doscientos sin la enfermedad; a pesar de la aparición de la penicilina en la década de los cuarenta y que fue probada como tratamiento seguro y eficaz contra esta enfermedad, los investigadores negaron el tratamiento y la información sobre el mismo a los participantes y continuaron su observación. El estudio continuó hasta 1972 cuando fue denunciado públicamente (3).

Después de conocerse lo sucedido en Tuskegee, el gobierno de los Estados Unidos creó en 1974 la Comisión Nacional para la Protección de los Sujetos Humanos de Investigación Biomédica y del Comportamiento, con el propósito de establecer la fundamentación ética que debía existir en toda investigación que involucrara seres humanos. En 1979 después de trabajar cuatro años, esta comisión produjo un documento conocido como el Informe Belmont (6).

En él se establecieron los principios éticos que servirían de base para formular directrices específicas y cuyo objetivo primordial era proteger a los sujetos de investigación. Estos principios son: respeto a las personas, beneficencia y justicia.

Estos principios tienen una aplicación práctica en el campo de la investigación propiamente dicha: la obtención del consentimiento informado entendido como un proceso mediante el cual la persona, recibiendo toda la información, con la comprensión adecuada y de forma voluntaria (sin coerción ni influencias indebidas) acepta participar libremente en una investigación. La valoración adecuada de los riesgos y beneficios, mediante un balance de la probabilidad de aparición de daños contra los posibles valores positivos para la salud o bienestar tanto del sujeto como de la comunidad y la selección justa de los sujetos, tanto a nivel individual como social, teniendo especial cuidado con las poblaciones vulnerables (6).

En 1982 el Consejo de las Organizaciones Internacionales de Ciencias Médicas (sigla en inglés CIOMS), publica las guías éticas internacionales para la investigación biomédica que involucra seres humanos, cuyo objetivo es ayudar a los investigadores e instituciones y a las autoridades sectoriales, regionales, nacionales e internacionales, a establecer y mantener normas para evaluación ética de los estudios epidemiológicos (7).

Se hace referencia, entre otros temas, al fortalecimiento de los comités de revisión tanto locales como nacionales, a las obligaciones de los patrocinadores de la investigación y se hace énfasis en la protección de los sujetos y a su aplicación en los países en vías de desarrollo que están definiendo sus propias pautas éticas (7).

Ezekiel Emanuel propone siete requisitos éticos que complementan los expuestos con anterioridad y que deben cumplir todos los protocolos de investigación. Estos requisitos deben ser tenidos en cuenta por investigadores, patrocinadores y miembros de los comités de ética de investigación y aunque tienen pretensión de universalidad y son justificados por valores y principios ampliamente reconocidos y aceptados, requieren una interpretación práctica y especificación dependiendo del contexto (8).

Estos requisitos incluyen: valor, validez científica $\mathbf{y}$ social, con resultados confiables y metodología adecuada; una selección equitativa de los sujetos y una proporción favorable de riesgo-beneficio tanto para el sujeto como para la comunidad. Asegurar la obtención correcta del consentimiento informado y el respeto a los sujetos inscritos, promoviendo la confidencialidad en el manejo de la información, la disposición de la información adecuada sobre datos relevantes nuevos y sobre los resultados de la investigación y el tratamiento oportuno de las reacciones adversas. Por último, el autor recalca la necesidad de la revisión independiente de los protocolos por parte de peritos idóneos que conformen los comités independientes que validen la responsabilidad social y se aseguren de respetar la dignidad del sujeto participante (8).

De la misma manera, Colombia ha implementado su propia reglamentación para proteger a los sujetos de investigación, con la Resolución número 008430 de 1993 , expedida por el entonces Ministerio de Salud: "por la cual se establecen las normas científicas, técnicas y administrativas para la investigación en salud" y resaltando que "la 
investigación que se realice en seres humanos deberá ser realizada por profesionales con conocimiento y experiencia para cuidar la integridad del ser humano" (9).

Ante la necesidad ética de valorar de manera independiente los protocolos de investigación en comités capacitados para dicho fin, la Organización Mundial de la Salud (OMS) publica en Ginebra en el año 2002 las Guías Operacionales para Comités de Ética que evalúan la investigación biomédica, y establece que "los estándares éticos y científicos para llevar a cabo investigación biomédica en humanos han sido desarrollados y establecidos en guías internacionales, incluyendo la Declaración de Helsinki, las Guías Éticas Internacionales para Investigación Biomédica que Involucra a Humanos del CIOMS ${ }^{1}$ y de la OMS, y las Guías para Buena Práctica Clínica, del ICH, publicadas en 1996. El cumplimiento de estas guías ayuda a asegurar que se promuevan la dignidad, derechos, seguridad y bienestar de los participantes en la investigación, y que los resultados de las investigaciones sean creíbles" (10). Estas guías sirven de orientación para la conformación de los comités, los procedimientos que deben desarrollar y las responsabilidades de los mismos.

De acuerdo con estas guías, los comités tienen como objetivo contribuir al desarrollo de la calidad y la consistencia en la evaluación ética de investigación biomédica, constituyen su parte primordial, y brindan protección a los pacientes que son sujetos de investigación. Definen el comité como un grupo multidisciplinario independiente, cuya responsabilidad es asegurar la protección de los derechos, dignidad, seguridad y bienestar de los seres humanos sujetos de investigación. Debe actuar en interés total de los participantes de la investigación y de las comunidades, tomando en consideración las leyes e instituciones regulatorias donde se lleva a cabo la investigación (10).

Los comités deben constituirse para llevar a cabo la revisión y evaluación competente de todos los aspectos éticos y metodológicos de los proyectos de investigación y deben asegurar que sus labores puedan desarrollarse libres de influencias que afecten su independencia. Estos comités deben tener una composición multidisciplinaria y sus integrantes tener conocimiento científico y ético relevante, con una distribución balanceada de género y edad, y además debe participar una persona natural que represente los intereses y asuntos de la comunidad (11).

También en Colombia con la Resolución 8430 se exigió la creación de un comité de ética de investigación en toda institución que realizara investigación biomédica y con

\footnotetext{
1. Pauta 2. "Todas las propuestas para realizar investigación en seres humanos deben ser sometidas a uno o más comités de evaluación científica y de evaluación ética para examinar su mérito científico y aceptabilidad ética. Los comités de evaluación deben ser independientes del equipo de investigación, y cualquier beneficio directo, financiero o material que ellos pudiesen obtener de la investigación, no debiera depender del resultado de la evaluación. El investigador debe obtener la aprobación o autorización antes de realizar la investigación. El comité de evaluación ética debiera realizar las revisiones adicionales que sean necesarias durante la investigación, incluyendo el seguimiento de su progreso".
}

la Resolución 3823 de 1997 se estableció que los proyectos de investigación en medicamentos deberían ser evaluados por el Instituto Nacional de Vigilancia de Medicamentos y Alimentos, Invima, que remitiría un informe trimestral al Ministerio de Salud, con copia de los resultados de dichos estudios, una vez concluidos; en el año 1999 Colciencias hace obligatorio que todos los proyectos de investigación que se presentan en las convocatorias del Programa Nacional de Ciencia y Tecnología de la Salud se acojan a la Resolución 8430 y sean evaluados y aprobados por un comité de ética de investigación (12).

Si bien es cierto que en Colombia no existían guías para la conformación y funcionamiento de los comités, en el año 2008 el Ministerio de la Protección Social promulga la Resolución 2378 "por la cual se adoptan las Buenas Prácticas Clínicas para las instituciones que conducen investigación con medicamentos en seres humanos" y que guía tanto la conformación y el funcionamiento de los comités como los comportamientos de los investigadores y de los patrocinadores que participan en cualquier investigación de medicamentos: "Toda investigación que recaiga sobre seres humanos debe ser evaluada y aprobada por el comité de ética institucional. El comité debe evaluar el proyecto de investigación, el formulario de consentimiento informado (documento que explica el objeto de la investigación, incluidos los riesgos y beneficios a los potenciales participantes), la información conocida sobre el fármaco (incluidos los informes de acontecimientos adversos inesperados) y toda publicidad potencial planificada para obtener participantes" (13).

Dentro de este marco histórico y normativo, a continuación se hará un recuento cronológico de las actividades del Comité de Ética de la Fundación Santa Fe de Bogotá, cuyas labores se remontan a 1983, con la misión permanente de proteger la dignidad y buscar el bienestar de los sujetos participantes en investigaciones.

\section{Comité de ética de investigación con seres humanos de la Fundación Santa Fe de Bogotá: más de 25 años de experiencia} Antecedentes institucionales y pertinencia de sus actividades

La Fundación Santa Fe de Bogotá, como entidad privada, desde su creación en 1972, ha tenido un claro compromiso por los aspectos humanos, éticos y sociales de la atención en salud y una permanente búsqueda de la excelencia y generación de conocimiento para contribuir a solucionar los problemas del sector y a mejorar la salud de las personas y la comunidad.

Desde sus inicios ha tenido un Comité de Credenciales, Ética Médica y conducta profesional, que actualmente es asesor de la junta directiva. De la misma manera, en 1983 se conforman el Comité de Ética de Investigación con Seres Humanos (CEI con SH); el Comité de Ética Hospitalaria y más recientemente la Unidad de Bioética. 
A finales de 2009 se constituye el Centro de Humanismo y Bioética, que también es asesor de la junta directiva y que, además de sus actividades propias, convoca y reúne a los Comités de Credenciales, Ética Médica y Conducta Profesional, de Ética Hospitalaria y de Ética en Investigación con Seres Humanos. En julio de 2010 se firma un compromiso de colaboración con la Organización Panamericana de la Salud (OPS) para desarrollar actividades conjuntas y de capacitación en el área de bioética. Como complemento de las actividades educativas, se establece un convenio con la Universidad de Miami en el mismo año. La fundación, en la actualidad, lidera la capacitación en ética de la investigación para todos los investigadores, no sólo de la institución sino de otros estamentos interesados en el tema, mediante el programa CITI (Collaborative Institutional Training Initiative) de la universidad en mención ${ }^{2}$.

\section{Antecedentes}

El comité se conformó inicialmente como Comité de Investigaciones Biomédicas en acta de septiembre de 1983 con los siguientes miembros: doctor Roberto Esguerra, doctor Efraín Otero, doctor Julio Portocarrero, doctor Hernando Sarasti y doctor Enrique Urdaneta "con la función de evaluar, aprobar o rechazar proyectos de investigación". En reuniones que se llevaban a cabo cada mes se establecieron las normas para presentar los protocolos de investigación y los parámetros para revisar tanto la parte metodológica como la ética.

A lo largo de los años otros profesionales del área de la medicina colaboraron y enriquecieron este comité como José Félix Patiño, Jaime Toro, Fernando Chalem, Alfonso Vargas, Jaime Herrera, Eduardo Castro, Guillermo López y Jaime Urdinola. Participaron de igual manera representantes de enfermería, de salud comunitaria, el director de la División de Educación, representantes del Ministerio de Salud, de la Escuela Colombiana de Medicina, de la Universidad del Bosque, de la Universidad de los Andes y un asesor de Colciencias. Se motivó la investigación dentro de la institución, estableciendo vínculos con la Universidad de los Andes para desarrollar seminarios sobre metodología; los estudios revisados incluían protocolos de la industria farmacéutica así como las investigaciones realizadas por los médicos y los estudiantes de la institución. A partir de 1987 el comité pasó a depender de la División de Educación y contaba con fondos propios aportados por parte de la institución y por Colciencias.

En 1988 se convirtió en Comité de Investigaciones Bio-

2. El Programa CITI (Collaborative Institutional Training Initiative) es un curso basado en Internet y desarrollado por voluntarios, que brinda material educacional sobre protección de sujetos humanos en investigaciones biomédicas y de conducta, buenas prácticas clínicas, conducta responsable de la investigación y trato humanitario a animales de laboratorio. Es un esfuerzo conjunto del Fred Hutchinson Cáncer Research Center y la Universidad de Miami. (LITEWKA, Sergio; GOODMAN, Kenneth y BRAUNSCHWEIGER, Paul. EL PROGRAMA CITI: UNA ALTERNATIVA PARA LA CAPACITACIÓN EN ÉTICA DE LA INVESTIGACIÓN EN AMÉRICA LATINA. Acta bioeth. [online]. 2008, vol.14, n.1, pp. 54-60) médicas y Publicaciones, con el fin de continuar revisando no sólo los protocolos de la industria farmacéutica y los trabajos de investigación de los miembros de la institución sino además de asesorar la producción del material científico que se publicaba en la institución (publicaciones, videos, libros o posters).

Durante estos años se implementó la capacitación de los miembros de la institución en aspectos metodológicos y estadísticos y se crearon las guías para evaluar el seguimiento de los procesos de los estudios y para los reportes finales de los mismos.

En el año de 1989 el comité participó en la elaboración del reglamento de investigación de productos farmacéuticos en conjunto con representantes del Ministerio de Salud. Paralelo a las actividades de revisión de los protocolos y los trabajos de investigación, se promovió la formación de comités de investigación en cada servicio de la Fundación, para estimular a los médicos institucionales, así como a los estudiantes, a realizar investigaciones.

En 1993, representantes de Colciencias y del Ministerio de Salud promocionaron el Programa de Ciencia y Tecnología, que contaba con recursos para promover la financiación de proyectos de investigación en el área biomédica y de tecnología y de esta manera ayudaron a fomentar la investigación en la Fundación. En este mismo año, la Fundación asumió los lineamientos de la Resolución 8430 emitida por el Ministerio de Salud, donde se establecían las normas científicas, técnicas y administrativas para la investigación en salud.

A partir de 1994, el comité trabajó con especial énfasis en la revisión de los consentimientos informados para cumplir la norma nacional expedida en 1993. Los miembros del comité recibieron capacitación en los aspectos relevantes que debería contener un consentimiento y en la manera como se debía obtener por parte de los investigadores, para proteger la integridad y la autonomía de los sujetos participantes en investigaciones.

Desde 1995 se decidió que los programas y proyectos de investigación fueran manejados por el Centro de Estudios e Investigación en Salud (CEIS), división que forma parte de la Fundación, y que el Comité dependiera de él. El Centro ofrecía asesoría metodológica y capacitación para promover la elaboración de trabajos de investigación en el área de salud pública y de las investigaciones clínicas.

En 1997 se modificó el proceso de nombramiento de los miembros del comité, no ya representantes de cada servicio de la Fundación, sino por la experiencia y el conocimiento en áreas específicas: ética, metodología y administración. Ya en este año y con esta conformación, el comité cumplía de manera más precisa con las recomendaciones internacionales para conformar los comités de revisión ética. Se inició de forma paralela y permanente la capacitación y reflexión sobre temas relacionados con la ética de investigación con seres humanos como la revisión de la Declaración de Helsinki, los principios rectores en investigación promulgados 
por el Informe Belmont y la resolución colombiana del año 1993.

Posteriormente en 1998, se aprobó un nuevo reglamento para el comité y éste adoptó el nombre de Comité Ético y Científico de Investigaciones y se prestó mucha atención al reporte de los efectos adversos producidos durante las investigaciones así como la exigencia de pólizas de seguros para asegurar la protección y el bienestar de los sujetos participantes. Se acató lo establecido en la Resolución 3823 de 1997 de la Comisión Asesora de Ciencia y Tecnología del Ministerio de Salud que facultaba al INVIMA a registrar y seguir todos los estudios de investigación.

Para este momento el comité había llegado a una madurez en su conformación y ejercicio de sus funciones con importantes avances en los procesos de revisión de los protocolos; de manera sistematizada se evaluaba la parte metodológica de los protocolos, todos los componentes necesarios en el consentimiento informado, el reporte cumplido de los efectos adversos, la seguridad de la existencia de las pólizas de cubrimiento y la idoneidad de todos los investigadores que llevaban a cabo las labores investigativas. Se instauraron tanto planillas y guías estructuradas para la revisión de los puntos enunciados así como manuales para la presentación adecuada de los protocolos al comité con todos los requisitos necesarios para poder llevar a cabo la revisión completa.

En el año 1999 participa en el comité por primera vez, de acuerdo con lo estipulado por la ley, un miembro representante de la comunidad. A partir de 2000 se adoptan las Guías de Buenas Prácticas Clínicas -Guías Tripartitas- elaboradas por la Conferencia Internacional de Armonización. En el año 2002 la dirección de la Fundación decidió fortalecer la investigación propia de la institución a través del CEIS y conformó un comité denominado Comité Corporativo de Ética de Investigación que cumpliendo las reglamentaciones internacionales y nacionales, se dedicaría casi exclusivamente a la revisión de los protocolos patrocinados por la industria farmacéutica.

En enero de 2007 inició su actividad como organismo independiente al considerar inconveniente la subordinación al CEIS, como ente encargado de la administración y coordinación de los protocolos de investigación. Buscando una total transparencia en su gestión, el comité quedó conformado por catorce miembros, con presupuesto propio y autonomía para deliberar y decidir. Sus objetivos quedaron estipulados de la siguiente manera:

- Velar por el cumplimiento de los objetivos y del buen funcionamiento del Comité.

- Evaluar los aspectos científicos, técnicos y éticos de todos los protocolos de investigación que recluten seres humanos.

- Evaluar la idoneidad de los profesionales investigadores.

- Hacer seguimiento y auditoría de la ejecución de los estudios

- Participar en las actividades programadas del Comité incluyendo aquellas relacionadas con capacitación.
A partir del año 2007, y de forma anual, se hace la inscripción de este comité ante la FDA y la Oficina para la Protección de los Sujetos de Investigación (en inglés. OHRP-Office for Human Research Protection del DHHS -Departamento de Salud y de Servicios Humanos- de los Estados Unidos) para poder contar con asesorías y consultas en casos especiales.

\section{Actividades y retos}

El comité en la actualidad realiza sus actividades de manera estructurada en reuniones quincenales. Está conformado por especialistas en metodología que revisan la pertinencia y rigor científico de los protocolos y por especialistas en ética que supervisan la selección justa de los sujetos, el balance de riesgos y beneficios, la autonomía de los sujetos participantes y la protección de los sujetos vulnerables. Cuenta además con la participación de un abogado que vigila el cumplimiento de los aspectos legales y de un miembro representante de la comunidad que protege los intereses de todos los sujetos por él representados.

El reto continúa siendo la evaluación y seguimiento adecuados de los trabajos de investigación que se desarrollan en la Fundación, con capacitación permanente de todos los miembros en temas relacionados con la investigación y los adelantos científicos para asegurar una labor profesional que vele por el cumplimiento de la misión del comité: la protección de la dignidad y del bienestar de los sujetos participantes en investigaciones.

\section{Conclusiones}

Este repaso histórico del surgimiento de los Comités de Ética de Investigación con Seres Humanos en el mundo sirve de marco para el recuento de las actividades del Comité de Ética de la Fundación Santa Fe de Bogotá. Así mismo, pone en evidencia su proceso de crecimiento a lo largo de estos años, no sólo a nivel de recurso humano sino también en la capacidad de evaluación de procesos y procedimientos. $\mathrm{Su}$ estructura le permite cumplir con excelencia la misión de proteger a los sujetos de investigación, respetando la misión y visión de la institución y acogiéndose a la normativa nacional e internacional vigente.

El comité en el ejercicio de sus funciones tiene clara su responsabilidad social de proteger a los participantes en investigación, velar por la validez social y científica de los protocolos revisados, sean de la industria farmacéutica o los productos de iniciativas investigativas de sus médicos institucionales y en la actualidad de los estudiantes de medicina y otros profesionales en formación.

Es importante recalcar el compromiso a lo largo de todos estos años de sus integrantes quienes, de manera desinteresada y profesional, han realizado todas las actividades de revisión metodológica y ética de los proyectos de investigación.

Hoy por hoy, es un comité que marca la pauta ante los estamentos reguladores nacionales (primer comité avalado formalmente por el INVIMA en 2010) y sirve de guía para 
otros comités similares. Es reconocido como uno de los más actualizados y organizados en su misión de proteger la dignidad, los derechos y el bienestar de los sujetos que participan en investigación, con una búsqueda permanente de conocimiento en todas las áreas científicas, pero sobre todo, comprometido por el bienestar social de la comunidad.

Este compromiso deberá extenderse a la búsqueda de escenarios de participación conjunta con miembros de otros comités en Bogotá y Colombia, con el fin de establecer conjuntamente lineamientos de evaluación metodológica y ética de los protocolos que se realizan en varias instituciones. De esta manera, al conformar redes institucionales se podría garantizar un mayor compromiso para fomentar investigaciones que respondan a las necesidades puntuales de nuestro país, para proteger las comunidades donde se llevan a cabo, para verificar la calidad y validez científica de las mismas y por último, para asegurar la transparencia e idoneidad de todas sus actividades.

\section{Referencias}

1. Gracia D. Investigación clínica 1. In: Edtorial el Buho, editor. Profesión Médica,investigación y justicia sanitaria. 1998.p.77-110.

2. Brussino Susana. Antecedentes y fundamentos de la ética de la investigación. Red Latinoamericana de Bioética. UNESCO. Primer curso de Ética de Investigación con Seres Humanos.Programa de Educación Permanente a Distancia. 1-33. 2006. Disponible en: www.redbioeticaunesco.org

3. Faden Ruth R, Beauchamp Tom L. The development of consent requirements in research ethics. In: Oxford University Press, editor. A history and theory of informed consent. Nueva York-Oxford: 1986.p.151-99.
4. Levine R. Nuremberg Code, Ethics and Regulation of Clinical Research. Second Edition ed. New Haven: 2010.

5. Asociación Médica Mundial. Declaración de Helsinki de la Asociación Médica Mundial. Principios éticos para las investigaciones médicas en seres humanos. 10-10-2008. Disponible en: www.cei.fmed.edu.uy/Declaracion_de_Helsinki_2008_ESPANOL.pdf

6. Comisión Nacional para la protección de los sujetos humanos de investigación biomédica y del comportamiento. Informe Belmont. Principios y guías éticos para la protección de los sujetos humanos de investigación. 2010. Disponible en: www. pcb.ub.edu/bioeticaidret/archivos/norm/InformeBelmont.pdf

7. Consejo de las Organizaciones Internacionales de Ciencias Médicas (CIOMS) Guías Éticas Internacionales para la Investigación Biomédica que involucra Seres Humanos. [Programa Regional de Bioética OPS/OMS]. 2-8-2010. Disponible en: www.paho.org/Spanish/BIO/CIOMS .

8. Emanuel E. ¿Qué hace que la investigación clínica sea ética? Siete requisitos éticos, pautas éticas de investigación en sujetos humanos: nuevas perspectivas Programa Regional de Bioética, Serie Publicaciones - 2003. Programa Regional de Bioética,Organización Panamericana de la Salud, Organización Mundial de la Salud OPS, OMS, editors. 2003. http://www.bioetica.ops-oms.org/

9. Normas científicas, técnicas y administrativas para la investigación en salud Resolución 8430 de 1993, República de Colombia.Ministerio de Salud, (1993). www.dib.unal.edu.co/promocion/etica_res_8430_1993.pdf

10. Guia Tripartita Armonizada de la Conferencia Internacional de Armonización.1996. Lineamientos para la Buena Práctica Clínica11. 5-9-2010. 5-9-2010. Disponible en: www.fda.gov/downloads/Drugs/.../Guidances/ucm073128.pdf

11. Organización Mundial de la Salud. Guías Operacionales para Comités de Ética que Evalúan Investigación Biomédica Organización Mundial de la Salud. Traducido por: Dr. James Zapata, Dr. Armando Torres y Dra. Dafna Feinholz-Klip. 2000. Disponible en: www.cba.gov.ar/imagenes/fotos/sal_serfis_guiaoms.pdf

12. Jaramillo M C. Comités de ética de investigación en humanos: el desafío de su fortalecimiento en Colombia 12. Biomédica, 138-144. 2006. Instituto Nacional de Salud.

13. Adopción oficial y con carácter obligatorio las Buenas Prácticas Clínicas para instituciones que conducen investigación con medicamentos en seres humanos. Resolución 2378 de 2008, República de Colombia.Ministerio de la Protección Social, (2008). 200.13.243.186/Docencia/docs/Decreto2378de2008.pdf 